\title{
The UK NCRI MAGIC Trial of Perioperative Chemotherapy in Resectable Gastric Cancer: Implications for Clinical Practice
}

\author{
Yu Jo Chua, MBBS, and David Cunningham, MD, FRCP
}

Department of Medicine, Royal Marsden Hospital, Surrey and London, United Kingdom

The 503-patient United Kingdom National Cancer Research Institute (NCRI) Medical Research Council Adjuvant Gastric Infusional Chemotherapy (MAGIC) trial is the first randomized trial to demonstrate a conclusive survival benefit from the use of perioperative chemotherapy for patients with resectable adenocarcinoma of the stomach, gastroesophageal junction, and lower esophagus, compared with surgery alone. ${ }^{1}$ In the intention-to-treat (ITT) analysis, patients who received perioperative chemotherapy with the ECF regimen (epirubicin, cisplatin, and continuous infusion 5-fluorouracil, 5FU) had a 5year survival of $36 \%$, compared with $23 \%$ in patients treated with surgery alone (hazard ratio for death, $0.75 ; 95 \%$ confidence interval, $0.60-0.93 ; \mathrm{P}=0.009$ ). Progression-free survival was also improved by perioperative chemotherapy (hazard ratio for progression, 0.66 ; 95\% confidence interval, $0.53-0.81$; $\mathrm{P}<$ $.001)$. These results support the use of this treatment strategy as an option for patients with resectable gastric cancer as an alternative to postoperative chemoradiotherapy, which has been shown in a previous randomized trial (the US Intergroup 0116 trial, INT 0116) to also be of benefit in these patients, and are expected to influence the standard treatment of this disease particularly in the UK and Europe. ${ }^{2}$

Received February 12, 2007; accepted March 14, 2007; published online: July 27, 2007.

Address correspondence and reprint requests to: David Cunningham, MD, FRCP; E-mail: david.cunningham@rmh.nhs.uk

Published by Springer Science+Business Media, LLC $\odot 2007$ The Society of Surgical Oncology, Inc.
The positive results of this trial are consistent with the results seen in several randomized trials of the ECF chemotherapy regimen in the advanced disease setting. ${ }^{3-6}$ Even though it was originally developed in the 1980s, this regimen has repeatedly been shown to be both efficacious and well tolerated and has not been bettered by modifications to the triplet combination. The main drawback of ECF is the continuous infusion $5 \mathrm{FU}$, which requires long-term intravenous access (e.g., Hickman's line or similar) and an infusion pump. These are associated with a risk of linerelated complications and inconvenience to patients.

The MAGIC trial study design and results have attracted several criticisms, many of which reflect difficulties inherent to the group of patients targeted by the trial or period during which the trial was open to recruitment (1994-2002). ${ }^{7-12}$ These include the lack of detailed preoperative local staging, the type of surgery performed, poor rates of commencement and completion of postoperative chemotherapy, and the limited ability to perform subgroup analyses on the results of the trial.

\section{LACK OF DETAILED PREOPERATIVE LOCAL STAGING}

Briasoulis and colleagues have lamented the lack of survival outcome data for patients in the MAGIC trial by disease stage. ${ }^{12}$ Citing previous reports of the excellent survival of the cohort of patients with resected gastric cancer who are lymph node negative 
on histopathology examination, the authors propose that this information may be useful in limiting the use of perioperative chemotherapy to patients with more locally advanced tumors while sparing those with early-stage disease from additional treatment as is the case with localized colon cancer. In contrast with good results seen with surgery alone in the Far East, it is questionable whether these outcomes can be generalized to patients in the West. For example, in a report from the US National Cancer Data Base on patients undergoing gastrectomy in 1985-1995, patients with TNM stage II disease were reported to have a 5 -year survival rate of only $34 \% .{ }^{13}$ Critics may attribute the poor outcome observed to suboptimal surgical technique, in particular with regard to the extent of lymph node dissection, but trials evaluating gastrectomies with so-called D1 and D2 lymph node dissections conducted in the West have not shown a survival advantage with more extensive surgery. ${ }^{14}$ In addition, an update of the results of US INT 0116 reported no significant interaction between $\mathrm{T}$ - or $\mathrm{N}$ stage and treatment outcome in an exploratory subgroup analysis. ${ }^{15}$ Considering the relatively poor outcome of patients with resected gastric tumors, even those with stage II disease, a worthwhile benefit from perioperative treatment can still be expected.

All of the published reports of the outcomes of patients with resected gastric cancer by stage have stratified patients using histopathological staging. While histopathology remains the gold standard for staging the extent of disease, its prognostic value in patients who have received preoperative treatment has not been validated, and its clinical utility is limited at the time of diagnosis (prior to resection), which is precisely when clinicians must decide between recommending a perioperative strategy or proceeding directly to surgery. Whereas endoscopic ultrasound (EUS) is proving to be increasingly useful for local staging of gastric tumors, its use and availability is not yet universal. Computed tomography (CT), while useful for excluding distant metastasis, is less accurate for local staging. Although now commonly available in the United Kingdom, EUS was not widely available when the MAGIC trial was conducted and so could not be included as a requirement of the study protocol. Our ability to tailor treatments to individual patient and disease requirements is limited, at least based on pretreatment staging methods available even today. This is undoubtedly an area of great importance and will become more clinically relevant as the treatment options available to these patients expands.

\section{IS POSTOPERATIVE TREATMENT REQUIRED?}

The difficulties of delivering postoperative treatment in patients undergoing gastrectomy are reflected in the fact that only $55 \%$ of the patients randomized to perioperative chemotherapy actually commenced treatment after surgery, and only $42 \%$ of the 250 completed all six cycles of protocol chemotherapy. ${ }^{1}$ Upper gastrointestinal surgery is a major invasive procedure often associated with a prolonged recovery, which is compounded by these patients being already debilitated by tumor-related symptoms or compromised by comorbidities. True to the aggressive nature of gastric cancer, early progression of disease is also a well-known problem. Indeed, the most common reason for not commencing postoperative treatment in the MAGIC trial was disease progression or early death. This aspect of the trial is one major difference from US INT 0116, in which patients were randomized between 20 and 40 days after curative surgery was performed, thus potentially selecting for a better patient cohort in terms of recovery from surgery and also for being free of early disease relapse. ${ }^{2}$ This is also the main reason that the results of the two trials should not be compared. In US INT 0116, 64\% of the 281 patients randomized to postoperative chemoradiotherapy completed protocol treatment, the predominant reason for early treatment cessation being treatment-related toxicity (17\% of 281).

A frequently raised question is whether given the survival improvement seen in MAGIC despite the poor rate of completion of protocol treatment, the benefit observed largely is from the three cycles of chemotherapy given preoperatively. A related question is whether postoperative treatment can be omitted altogether. Although tempting, performing an analysis to address these issues would be inappropriate as it is likely to be heavily biased. For example, restricting an analysis for survival to only the cohort of patients who completed all six cycles of treatment would select for patients predisposed toward a better outcome for reasons other than the efficacy of treatment since many of the patients not included would have been excluded for disease-related factors.

\section{THE LIMITATIONS OF SUBGROUP ANALYSES}

The MAGIC trial was powered to show survival benefit in the overall treatment cohort and was not designed with the expectation that significant results 
would be found for any of the subgroups of patients, nor would it have been practical to increase recruitment to allow for such subgroup analyses. ${ }^{1,16}$ As it is, the trial took almost 8 years to complete accrual. Similarly, US INT 0116 recruited 556 eligible patients over a 7-year period, both trials illustrating the challenge recruiting patients to these type of clinical trials. ${ }^{2}$ Indeed, a Dutch trial of preoperative chemotherapy that was initiated at the same time as MAGIC only recruited 59 patients over 2.5 years and was closed prematurely when an early interim analysis prompted by the slow accrual found the trial was unlikely to meet its objective of improving the curative resection rate. ${ }^{17}$

Any subgroup analyses performed within the MAGIC trial should be exploratory only and should certainly not be the basis for recommending standard treatments, whether on the basis of stage grouping, or with regard to the need for postoperative chemotherapy. The ITT analysis performed is the more appropriate estimate of the overall effect of treatment. ${ }^{16,18}$

\section{THE NEXT RANDOMIZED TRIAL OF PERIOPERATIVE TREATMENT: UK NCRI ST03}

The follow-on study for MAGIC, to be known as the UK NCRI ST03 trial, is expected to open to accrual in the second quarter of 2007. This trial, which will be UK-led, will be open to the participation of international sites and aims to recruit a total of 1100 patients with resectable adenocarcinoma of the stomach or gastroesophageal junction (Siewert type III only) who will be randomized to perioperative chemotherapy with ECX (epirubicin, cisplatin, and capecitabine) or the same plus the antivascular endothelial growth factor (VEGF) monoclonal antibody bevacizumab. ECX has been chosen as the chemotherapy for this study because of the convenience of the oral administration of capecitabine, which replaces the $5 \mathrm{FU}$ and the associated requirement for intravenous lines and infusion pumps. It is hoped this will improve the acceptability of treatment to patients. The results of the recently reported REAL2 advanced disease trial have also shown that this substitution can be made without compromising the efficacy or safety of the regimen. ${ }^{6}$ Bevacizumab is a targeted agent that has proven benefit in several solid tumor types including colorectal, breast, and non-small cell lung cancer. A phase II trial in gastric cancer with cisplatin and irinotecan has reported promising results. ${ }^{19}$ The benefits of using bevacizumab in the context of perioperative chemotherapy may be to improve the response rates to chemotherapy and to more effectively treat micrometastatic disease, which is undoubtedly the cause of disease relapse in these patients despite apparently complete resections.

Because of the effects of VEGF on angiogenesis, an important component of the wound healing process, there is a theoretical risk of increased perioperative complications with the addition of bevacizumab to treatment. A mandatory interval of 8 weeks between the last dose of bevacizumab and surgery is specified in the protocol (equivalent to 5-6 weeks from the last dose of capecitabine) to minimize this risk. Similarly, postoperative bevacizumab is not recommenced until a minimum of 6 weeks has elapsed. As there have been no direct trials evaluating the effects of antiVEGF treatment on perioperative complications, these intervals were derived from experience from the randomized trials already conducted and on expert opinion. ${ }^{20,21}$ It is noteworthy that similar breaks from treatment were specified in the MAGIC trial so that in fact these recommendations will not amount to a change from current practice.

\section{CONCLUSIONS-IMPLICATIONS FOR STANDARD PRACTICE}

Based on the results of MAGIC, perioperative chemotherapy with ECF (or ECX) should be considered a standard treatment option for patients with resectable gastric cancer. There is, however, no evidence to suggest that either perioperative chemotherapy or postoperative chemoradiotherapy is superior. A significant factor that will likely determine the preferred strategy is the referral patterns that are prevalent in a given center. For perioperative chemotherapy to be feasible, patients must be referred to an oncologist prior to surgery so that consideration can be given to this strategy. In the United Kingdom, this management pathway is well suited to the requirement that the management of all new patients is discussed by an appropriately qualified multidisciplinary team, in this case consisting of specialist oncologists, surgeons, gastroenterologists, radiologists, and pathologists.

\section{REFERENCES}

1. Cunningham D, Allum WH, Stenning SP, et al. Perioperative chemotherapy versus surgery alone for resectable gastroesophageal cancer. $N$ Engl J Med 2006; 355:11-20. 
2. Macdonald JS, Smalley SR, Benedetti J, et al. Chemoradiotherapy after surgery compared with surgery alone for adenocarcinoma of the stomach or gastroesophageal junction. $N$ Engl J Med 2001; 345:725-30.

3. Waters JS, Norman A, Cunningham D, et al. Long-term survival after epirubicin, cisplatin and fluorouracil for gastric cancer: results of a randomized trial. Br J Cancer 1999; 80:269 72.

4. Webb A, Cunningham D, Scarffe JH, et al. Randomized trial comparing epirubicin, cisplatin, and fluorouracil versus fluorouracil, doxorubicin, and methotrexate in advanced esophagogastric cancer. J Clin Oncol 1997; 15:261-7.

5. Ross $\mathrm{P}$, Nicolson M, Cunningham D, et al. Prospective randomized trial comparing mitomycin, cisplatin, and protracted venous-infusion fluorouracil (PVI 5-FU) With epirubicin, cisplatin, and PVI 5-FU in advanced esophagogastric cancer. $J$ Clin Oncol 2002; 20:1996-2004.

6. Cunningham D, Rao S, Starling N, et al. Randomised multicentre phase III study comparing capecitabine with fluorouracil and oxaliplatin with cisplatin in patients with advanced oesophagogastric (OG) cancer: The REAL 2 trial. J Clin Oncol 2006; 24:abstr 4017.

7. Harewood GC. Treatment of gastric cancer. $N$ Engl J Med 2006; 355:1386; author reply $1387-8$.

8. Fielding J, Peake D. Treatment of gastric cancer. $N$ Engl J Med 2006; 355:1386-7; author reply $1387-8$

9. Singh J, Williamson SK, Malani AK. Treatment of gastric cancer. $N$ Engl J Med 2006; 355:1386; author reply 1387-8.

10. Boot H, Jansen EP, Cats A. Treatment of gastric cancer. $N$ Engl J Med 2006; 355:1387; author reply 1387-8.

11. Jani K. Treatment of gastric cancer. $N$ Engl J Med 2006; $355: 1387$; ; author reply $1387-8$.

12. Briasoulis E, Fatouros M, Roukos DH, Kappas AM. Level I evidence in support of perioperative chemotherapy for operable gastric cancer: sufficient for wide clinical use? Ann Surg Oncol 2007; (in press).
13. Hundahl SA, Phillips JL, Menck HR. The National Cancer Data Base Report on poor survival of U.S. gastric carcinoma patients treated with gastrectomy: Fifth Edition American Joint Committee on Cancer staging, proximal disease, and the "different disease" hypothesis. Cancer 2000; 88:921-32.

14. Cuschieri A, Weeden S, Fielding J, et al. Patient survival after D1 and D2 resections for gastric cancer: long-term results of the MRC randomized surgical trial. Surgical Co-operative Group. Br J Cancer 1999; 79:1522-30

15. Macdonald JS, Smalley S, Benedetti J, et al. Postoperative combined radiation and chemotherapy improves disease-free survival (DFS) and overall survival (OS) in resected adenocarcinoma of the stomach and gastroesophageal junction: Update of the results of Intergroup Study INT-0116 (SWOG 9008). Program and Proceedings of the 2004 Gastrointestinal Cancers Symposium, San Francisco, CA: abstr 6.

16. Cunningham D, Allum WH, Stenning SP. Treatment of gastric cancer. $N$ Engl J Med 2006; 355:1387-8.

17. Hartgrink HH, van de Velde CJ, Putter H, et al. Neo-adjuvant chemotherapy for operable gastric cancer: long term results of the Dutch randomised FAMTX trial. Eur J Surg Oncol 2004; 30:643-9.

18. Yusuf S, Wittes J, Probstfield J, Tyroler HA. Analysis and interpretation of treatment effects in subgroups of patients in randomized clinical trials. JAMA 1991; 266:93-8.

19. Shah MA, Ramanathan RK, Ilson DH, et al. Multicenter phase II study of irinotecan, cisplatin, and bevacizumab in patients with metastatic gastric or gastroesophageal junction adenocarcinoma. J Clin Oncol 2006; 24:5201-6.

20. Scappaticci FA, Fehrenbacher L, Cartwright T, et al. Surgical wound healing complications in metastatic colorectal cancer patients treated with bevacizumab. J Surg Oncol 2005; 91:17380.

21. Ellis LM, Curley SA, Grothey A. Surgical resection after downsizing of colorectal liver metastasis in the era of bevacizumab. J Clin Oncol 2005; 23:4853-5. 